\title{
Simultaneous Bilateral Anterior and Posterior Lenticonus without Renal Association - Case Report
}

\author{
Sowmya Raveendra Murthy* and Nitya Raghu \\ Department of Pediatric Ophthalmology and Strabismus, Sankara Eye Hospital, \\ Bengaluru, India \\ *Corresponding Author: Sowmya Raveendra Murthy, Department of Pediatric \\ Ophthalmology and Strabismus, Sankara Eye Hospital, Bengaluru, India.
}

Received: April 17, 2020

Published: June 01, 2020

(C) All rights are reserved by Sowmya

Raveendra Murthy and Nitya Raghu.

\begin{abstract}
Aims: To report a case of bilateral anterior and posterior lenticonus with sensorineural hearing loss, without renal associations. Case Report: We describe a case of bilateral anterior and posterior lenticonus associated with sensorineural hearing loss and absence of family history. No renal association could be identified on urine routine and microscopy or ultrasound abdomen. Clear lens extraction with posterior chamber intraocular lens (PCIOL) was performed for the patient.

Conclusion: The presence of bilateral anterior and posterior lenticonus is rare but may be a presentation of Alport syndrome. Clear lens extraction with intraocular lens is a viable treatment option with good visual prognosis.

Keywords: Anterior Lenticonus; Posterior Lenticonus; Alport Syndrome; Nephritis
\end{abstract}

\section{Abbreviations}

OD: Right Eye; OS: Left Eye

\section{Introduction}

Lenticonus is a rare condition resulting in conical protrusion of the lens capsule, most commonly anterior, along with lens cortex. This change in the shape of the lens is the cause for irregular astigmatism and spherical aberrations, causing significant vision loss. Presence of bilateral simultaneous anterior and posterior lenticonus is rare and there have been very few case reports in literature.

Anterior lenticonus is most commonly associated with Alport syndrome, a genetic disorder known to affect collagen type IV. It is associated with anterior lenticonus, sensorineural hearing loss and nephritis, presenting as progressive hematuria [1]. Other ocular associations are posterior polymorphous corneal dystrophy, cataract, peripheral retinal thinning, or dot fleck retinopathy.

In the absence of other associated pathologies causing vision loss, clear lens extraction with posterior chamber intraocular lens is known to give good results [2-4]. Possible difficulties could be during rhexis due to the thin anterior capsule resulting in peripheral run offs on attempting continuous curvilinear capsulorhexis (CCC).
We present a case with bilateral anterior and posterior lenticonus in a young Indian male without renal associations who benefitted from clear lens extraction with PCIOL.

\section{Case Report}

A 30 year old male presented to us with gradual painless diminution of vision in both eyes for the past $5-6$ years. He gave no history of use of glasses in the past or any other visual symptoms. He also suffered from sensorineural hearing loss which gradually worsened around the age of 10 - 12 years. There was no family history of similar complaints. He had no history of hematuria.

On examination, his unaided vision was 3/60 and 6/60 in right and left eyes respectively. On slit lamp biomicroscopy, there was conical protrusion of anterior lens surface in the centre with a distinct 'oil droplet sign' on retroillumination (Figure 1).

On careful examination, mild conical protrusion of posterior surface of lens bilaterally was also noticed, though not as prominent as the anterior lens surface (Figure 2 and 3). The lens showed no cataractous changes. Fundus examination showed mild arteriolar attenuation. Remaining examination was unremarkable.

Dilated retinoscopy was done from the periphery of the lens as the lenticonus prevented neutralisation of the reflex in the centre. 


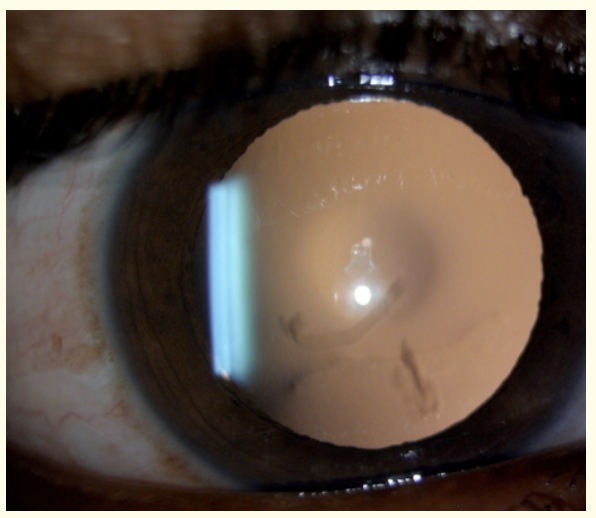

Figure 1: Classic 'oil droplet sign' on retroillumination seen in the right eye.
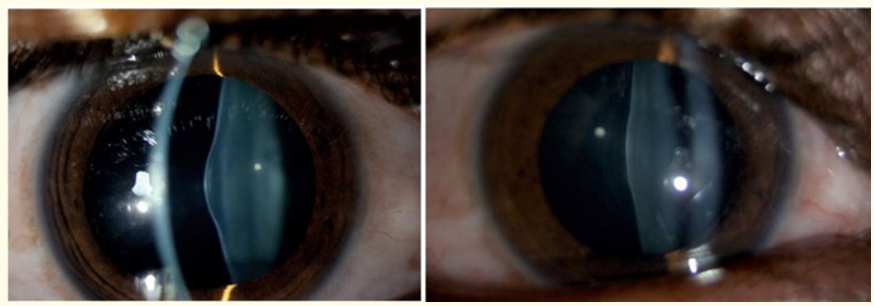

Figure 2: Slit lamp photograph showing the anterior lenticonus and posterior lenticonus in the right eye.
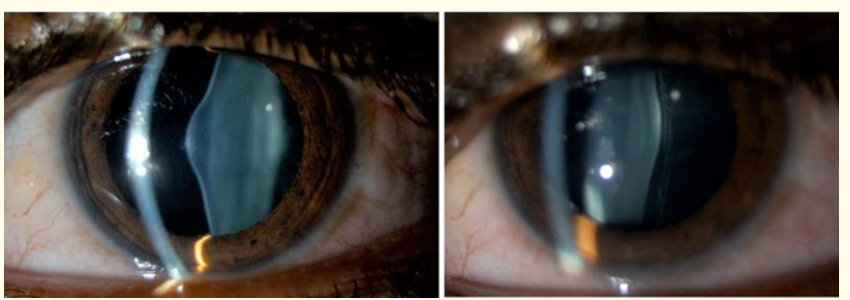

Figure 3: Slit lamp photograph showing the anterior lenticonus and posterior lenticonus in the left eye.

Subjective refraction showed a slight improvement to (OD) 6/36 +4.00DS/-0.50DC at $120 \mathrm{deg}$ and (OS) 6/60 +4.00DS/-0.50DC at $180 \mathrm{deg}$.

Investigations were ordered to rule possible renal associations. Serum creatinine was $1.1 \mathrm{mg} / \mathrm{dl}$ and Urine routine, microscopy was normal with no evidence of blood cells in urine. An ultrasound abdomen was carried out to look for renal associations but was found to be normal
Considering the poor vision and no improvement with refractive correction, a clear lens extraction of right eye with a posterior capsular intraocular lens was planned. Intraoperatively, the anterior capsule felt brittle, not elastic as was expected. Continuous curvilinear capsulorhexis was attempted using rhexis forceps but multiple secondary rhexis had to be done to get an adequate sized rhexis.

Posterior capsule didn't show any thinning. IOL was placed in bag. Patient was doing well on post op day 1 with a vision of 6/9.

\section{Results and Discussion}

Lenticonus occurs due to weakening in the anterior or posterior lens capsule. Alport is known to be associated with anterior lenticonus; posterior lenticonus is seen more commonly as an isolated finding or in Lowe's syndrome. Alport syndrome occurs due to a mutation in COL4A1-6 genes which is responsible for collagen IV synthesis, the predominant collagen in any basement membrane. This leads to lens capsular weakening, and similarly affects the basilar membrane of the organ of Corti and glomerulus, causing sensorineural hearing loss and hematuria respectively. The patients mostly develop microscopic hematuria by $6-7$ years of age progress to renal failure by age 20 [5]. Sensorineural hearing loss is also known to progress in the early second decade of life. Lenticonus develops only in the second decade and hence is not associated with amblyopia [5]. Alport has been associated with other corneal and retinal pathologies as well, including retinal thinning and dotfleck retinopathy.

Our patient presented had simultaneous anterior and posterior lenticonus and sensorineural hearing loss with no evidence of nephritis. He had mild arteriolar attenuation with no other causative signs in the fundus. The absence of nephritis in our patient cannot be explained but may develop manifestations later in life.

There have been few reports of simultaneous anterior and posterior lenticonus in patients of Alport presenting with other associated conditions like sensorineural hearing loss, dot-fleck retinopathy or hematuria [6]. Gupta., et al. [5] presented four such cases of anterior and posterior lenticonus with varying systemic manifestations.

M Hima Bindu [7] has reported a case of a 21 year old male with simultaneous anterior, and posterior lenticonus with no other systemic manifestation, similar to our case except for the presence of hearing loss in our patient. 
Ravi Kant., et al. [1] reported a case of a 22 year old female presenting with only combined lenticonus and hearing loss with no renal manifestations, with only microscopic hematuria being noted. It has also been speculated that absence of nephritis, though rare, could mean delayed systemic presentation and that it is imperative to pick up the diagnosis for close follow up in the future [7]. This might help delay the onset of end stage renal failure [1].

Due to the lenticular astigmatism and high spherical aberrations in patients with lenticonus, visual acuity is poor and clear lens phacoemulsification is the only option to improve vision. There have been many reports in literature showing good visual outcome following this surgery.

Bayar., et al. [4] did a large scale study of phacoemulsification in 23 eyes of 15 patients with anterior lenticonus and achieved good post op results. Similarly, good post op vision was achieved by Joseph D., et al. [8] and Sukhija., et al. [2] who operated on combined anterior and posterior lenticonus in Alport.

Gupta., et al. [5] operated on 6 eyes with simultaneous anterior and posterior lenticonus in Alport syndrome and 1 eye with ruptured anterior capsule. The cases were done under general anaesthesia due to loss of hearing of the patients. The common complication faced by them was difficulty in anterior capsulorhexis.

The anterior capsule in lenticonus is thinner and highly elastic and numerous techniques like starting rhexis from mid-periphery and tearing pattern like a 'cogwheel ripping', has been described by Sukhija., et al. and Boss., et al $[2,8]$. Ultrastructural vertical dehiscences have been documented on electron microscopy by Bayar., et al. [4] and this increases the chances of rhexis run offs. The need for careful hydrodissection in the presence of posterior capsule thinning and to avoid posterior capsule polishing has also been suggested [5].

We took up the case of clear lens phacoemulsification under local anaesthesia with peribulbar block. Capsulorhexis was done using rhexis forceps. Unlike other studies who had frequent run offs because of the highly elastic thin capsule [4,5], we found it difficult to make an adequate sized capsule because of its brittle nature. Rhexia was started from mid periphery and multiple secondary rhexis was done to get an adequate sized rhexis. Posterior capsule was intact. No complications were faced intraoperatively and PCIOL was placed in bag. Patient had a vision of 6/9 on first post-operative day. We have advised close follow ups for possible renal manifestations in the future.

\section{Conclusion}

The case re- emphasises the simultaneous occurrence of anterior and posterior lenticonus in patients with Alport's syndrome. Clear lens extraction with IOL implantation improves vision in these patients.

\section{Conflict of Interest}

No financial interest or any conflict of interest exists.

\section{Bibliography}

1. Bamotra Ravi Kant. "Simultaneous Bilateral Anterior and Posterior Lenticonus in Alport Syndrome". Journal of Clinical and Diagnostic Research 11.8 (2017): ND01-ND02.

2. Sukhija Jaspreet., et al. "Phacoemulsification and Intraocular Lens Implantation in an Alport's Syndrome Patient with Bilateral Anterior and Posterior Lenticonus". Journal of Cataract and Refractive Surgery 29.9 (2003): 1834-1836.

3. Sonarkhan S., et al. "Bilateral Anterior Lenticonus in a Case of Alport Syndrome: A Clinical and Histopathological Correlation after Successful Clear Lens Extraction". BMJ Case Reports (2014): bcr2013202036.

4. Bayar Sezin Akca., et al. "Clear Lens Phacoemulsification in Alport Syndrome: Refractive Results and Electron Microscopic Analysis of the Anterior Lens Capsule". European Journal of Ophthalmology 24.3 (2014): 345-351.

5. Gupta Arvind., et al. "Clear Lens Extraction in Alport Syndrome with Combined Anterior and Posterior Lenticonus or Ruptured Anterior Lens Capsule". Journal of Cataract and Refractive Surgery 37.11 (2011): 2075-2078.

6. Tiwari Uma Sharan., et al. "Bilateral Combined Anterior and Posterior Lenticonus in Alport's Syndrome”. Romanian Journal of Ophthalmology 62.3 (2018): 228-230.

7. M Hima Bindu. "Bilateral simultaneous anterior and posterior lenticonus without systemic signs". Journal of Evidence Based Medicine and Healthcare 2.8 (2015): 1081-1085.

8. Boss Joseph D and Mark McDermott. "Capsulorhexis Tearing Pattern during Phacoemulsification in Anterior Lenticonus Due to Alport Syndrome". European Journal of Ophthalmology 26.3 (2016): e39-e41. 
Assets from publication with us

- Prompt Acknowledgement after receiving the article

- Thorough Double blinded peer review

- Rapid Publication

- Issue of Publication Certificate

- High visibility of your Published work

Website: www.actascientific.com/

Submit Article: www.actascientific.com/submission.php

Email us: editor@actascientific.com

Contact us: +919182824667 